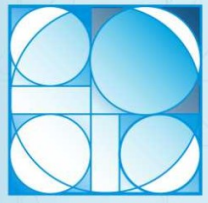

SBBq online symposium
Journal of Biochemistry Education

Revista de Ensino de Bioquímica

Revista de Enseñanza de Bioquímica

64 Sociedade Brasileira de Bioquimica
V. 20, N. 2 / 2020

Submitted in:4 dec 2020 Published in: 1 feb 2021

ISSN: 2318-8790

ISSN (until 2012): 1677-2318

\title{
Caracterização experimental de carboidratos
}

\author{
Experimental characterization of carbohydrates
}

\author{
Paula Fernandes Gáspari', Celene Fernandes Bernardes ${ }^{2}$ \\ ${ }^{1}$ Instituição: Prefeitura Municipal de Sumaré; ${ }^{2}$ Instituição: PUC-Campinas ${ }^{1}$ \\ 2 e-mail: celenefb@gmail.com
}

\begin{abstract}
Resumo
O estudo da caracterização molecular e propriedades químicas de carboidratos é tema constituinte dos planos de ensino das disciplinas de Bioquímica, nos cursos de Química, Biologia e Medicina. As aulas práticas foram realizadas de forma remota e com adaptações dos experimentos previstos para serem desenvolvidos em laboratório. As reações de Molisch, Fehling e Lugol, utilizadas para identificação e caracterização de carboidratos, foram realizadas de forma demonstrativa, com simulação das técnicas e dos resultados dos experimentos, após a explicação dos fundamentos teóricos. Foram utilizados os programas Paint e PowerPoint para desenhar os instrumentos e as etapas dos experimentos. A aula foi apresentada utilizando a ferramenta de animação do PowerPoint e gravada em vídeo. A aceitação dos alunos foi positiva embora tenham demonstrado a vontade de vivenciar as aulas no laboratório, principalmente para o desenvolvimento de habilidades no manuseio dos instrumentos.
\end{abstract}

Palavras-chave: carboidratos; aula remota; bioquímica experimental

\begin{abstract}
The study of the molecular characterization and chemical properties of carbohydrates is a constituent theme of the teaching plans at Biochemistry disciplines, in Chemistry, Biology and Medicine bachelor. The practical classes had been carried out remotely are adaptations of the experiments planned to be developed at the laboratory. The reactions of Molisch, Fehling and Lugol, used for the identification and characterization of carbohydrates, were demonstrate by schematic simulation of the techniques and the results of the experiments, after the explanation of the theoretical foundations. The Paint and PowerPoint software were used to design the instruments and the stages of the experiments. The class was presented using the PowerPoint animation tool and recorded on video. The students' acceptance was very good; although they demonstrated the willingness to experience the classes in the laboratory, mainly for the development of skills in handling the instruments.
\end{abstract}

Keywords: carbohydrates; remote class; experimental biochemistry 
Ficha da aula

\begin{tabular}{|c|c|}
\hline Título & $\begin{array}{l}\text { Caracterização experimental de estruturas e propriedades químicas de } \\
\text { carboidratos }\end{array}$ \\
\hline Tipo & Prática ( x ) \\
\hline Público-alvo & Alunos das Faculdade de Química, Biologia e Medicina \\
\hline $\begin{array}{l}\text { Disciplinas } \\
\text { correlatas }\end{array}$ & $\begin{array}{l}\text { Bioquímica aplicada à Química - } 4^{\circ} \text { período do Curso de Química } \\
\text { Bioquímica Estrutural }-3^{\circ} \text { período do Curso de Ciências Biológicas } \\
\text { Bioquímica } \mathrm{A}-1^{\circ} \text { período do Curso de Medicina }\end{array}$ \\
\hline $\begin{array}{l}\text { Objetivos } \\
\text { educacionais }\end{array}$ & Estudo das estruturas e propriedades químicas de carboidratos. \\
\hline Justificativa & $\begin{array}{l}\text { O estudo deste conteúdo possibilitará: } \\
\text { - Ilustrar e aprofundar conhecimentos teóricos relacionados às estruturas e } \\
\text { propriedades químicas dos carboidratos; } \\
\text { - Identificar os diferentes carboidratos encontrados nos alimentos e os que são } \\
\text { metabolizados nas células } \\
\text { - Simular habilidades no manuseio dos instrumentos utilizados em laboratório } \\
\text { de bioquímica. }\end{array}$ \\
\hline $\begin{array}{l}\text { Conteúdos } \\
\text { trabalhados }\end{array}$ & $\begin{array}{l}\text { Estruturas e propriedades químicas das moléculas de carboidratos. } \\
\text { Identificação de carboidratos em amostras diversas. } \\
\text { Caracterização de carboidratos redutores e de polissacarídeos e identificação } \\
\text { em amostras diversas. }\end{array}$ \\
\hline Duração & 2 horas/aula por curso. \\
\hline $\begin{array}{l}\text { Materiais } \\
\text { usados }\end{array}$ & $\begin{array}{l}\text { Programa de computador Paint e PowerPoint para a aula na forma remota. } \\
\text { Materiais necessários para a aula presencial: } \\
\text { Soluções aquosas de carboidratos glicose, frutose, maltose, sacarose e amido } \\
\text { na concentração de } 1 \mathrm{~g} \% \text {. } \\
\text { Reativos de Molisch, Fehling e Lugol. } \\
\text { Ácido sulfúrico concentrado puro para análise. } \\
\text { Tubos de ensaio, banho-maria fervente e pipetas. }\end{array}$ \\
\hline
\end{tabular}




\section{Introdução}

O estudo do tema de carboidratos é essencial para a disciplina de Bioquímica considerando que a energia celular é obtida, em grande parte, a partir do metabolismo dos monossacarídeos [1]. A ingestão dos carboidratos ocorre com as moléculas em diferentes formas químicas estruturais e graus de complexidade, como polissacarídeos, oligossacarídeos, dissacarídeos e monossacarídeos [2].

Nesse contexto, o ensino da bioquímica de carboidratos abrange aspectos químicos estruturais e metabólicos, permeando pelos mecanismos de digestão enzimática para transformação de carboidratos complexos em monossacarídeos simples e absorção celular. O tema é normalmente desenvolvido em aulas teóricas e práticas laboratoriais.

Neste ano de 2020, com a necessidade de desenvolver aulas remotas e não presenciais, as práticas laboratoriais foram adaptadas. Com a utilização de tecnologia simples e os programas "Paint" e "PowerPoint", os instrumentos de laboratório foram desenhados e os experimentos das aulas práticas foram simulados, seguindo as etapas previstas para serem desenvolvidas no laboratório.

Os alunos avaliaram as aulas de forma positiva. O desenvolvimento do conteúdo prático possibilitou alcançar os objetivos de aprofundar conhecimentos e ilustrar os conceitos desenvolvidos teoricamente. Entretanto, foi unânime a ponderação da "vontade" de realmente "fazer" os experimentos e vivenciar o laboratório.

A utilização de aulas práticas simuladas foi um importante instrumento, no momento atual, de aulas remotas, mas não substitui as aulas experimentais em laboratório, principalmente pela não possibilidade de desenvolver as habilidades no manuseio dos instrumentos e de não permitir a "descoberta" dos resultados.

\section{Descrição da aula remota de caracterização de carboidratos}

A primeira etapa da aula compreende a indicação dos experimentos que serão realizados, conforme esquema apresentado na figura 1. 
Figura 1: Experimentos propostos para a aula

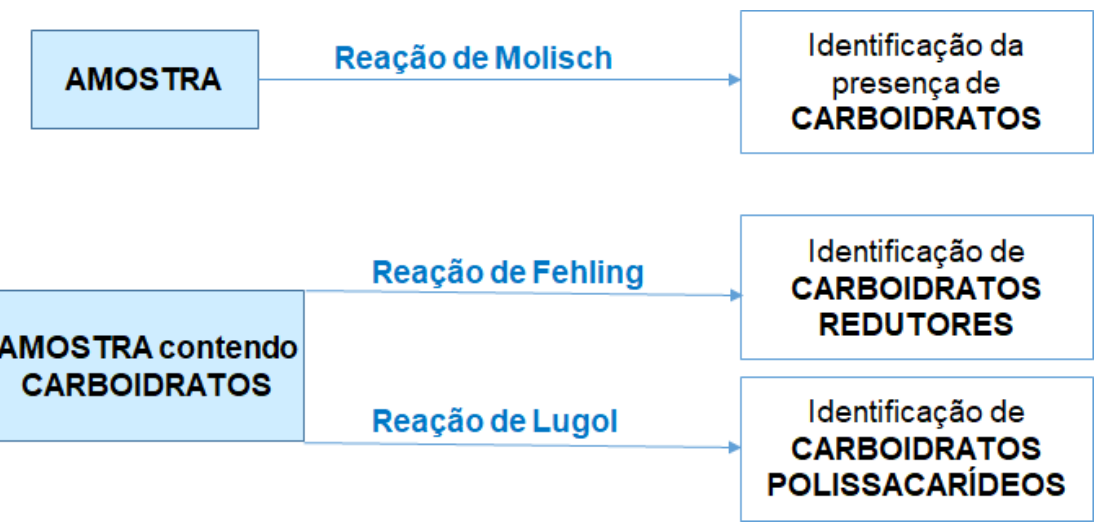

Figura 1: Experimentos propostos para a aula Fonte: autoria própria

Em seguida, utilizando a função "animação" do PowerPoint foram explicados os fundamentos teóricos, os procedimentos técnicos e os resultados de cada experimento [35].

Os procedimentos experimentais compreendem a identificação de 9 tubos de ensaio onde serão pipetados os volumes indicados das soluções de padrões, de amostras e de reagentes específicos para cada reação. Os esquemas dos experimentos e uma foto com os resultados característicos das reações de Molisch, Fehling e Lugol estão representados nas figuras 2, 3 e 4 .
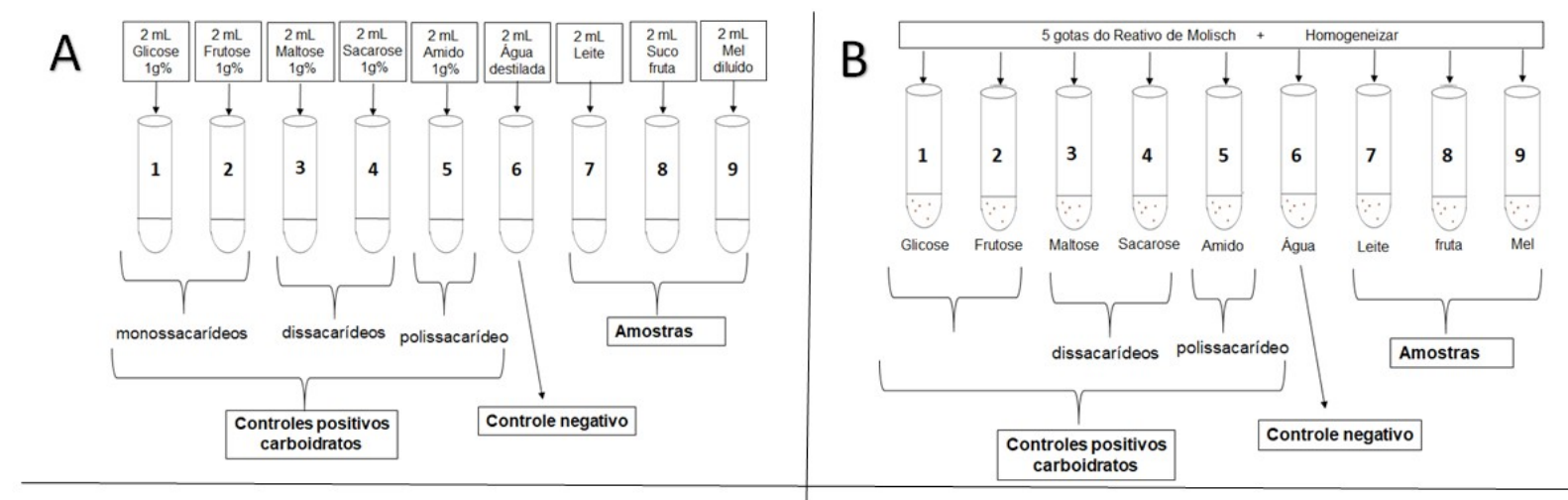

C

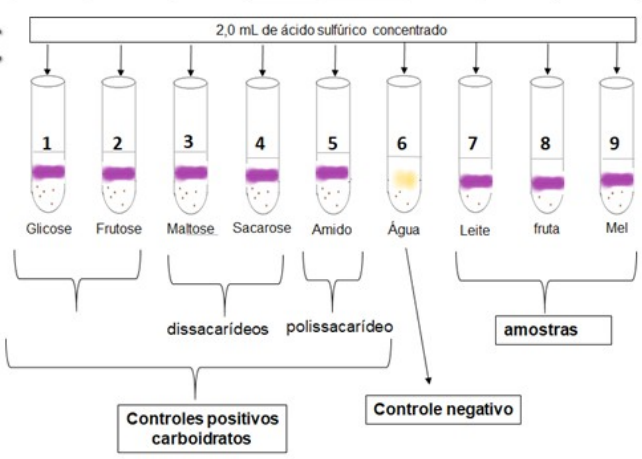

Figura 2: Reação de Molisch Fonte: autoria própria
$\mathrm{D}$

Resultados caracteristicos

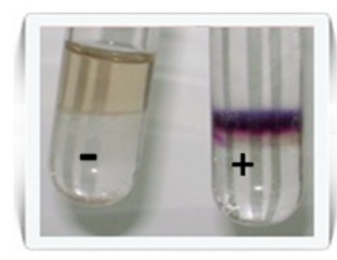

Conclusão: as amostras de leite, fruta e mel contêm carboidratos. 


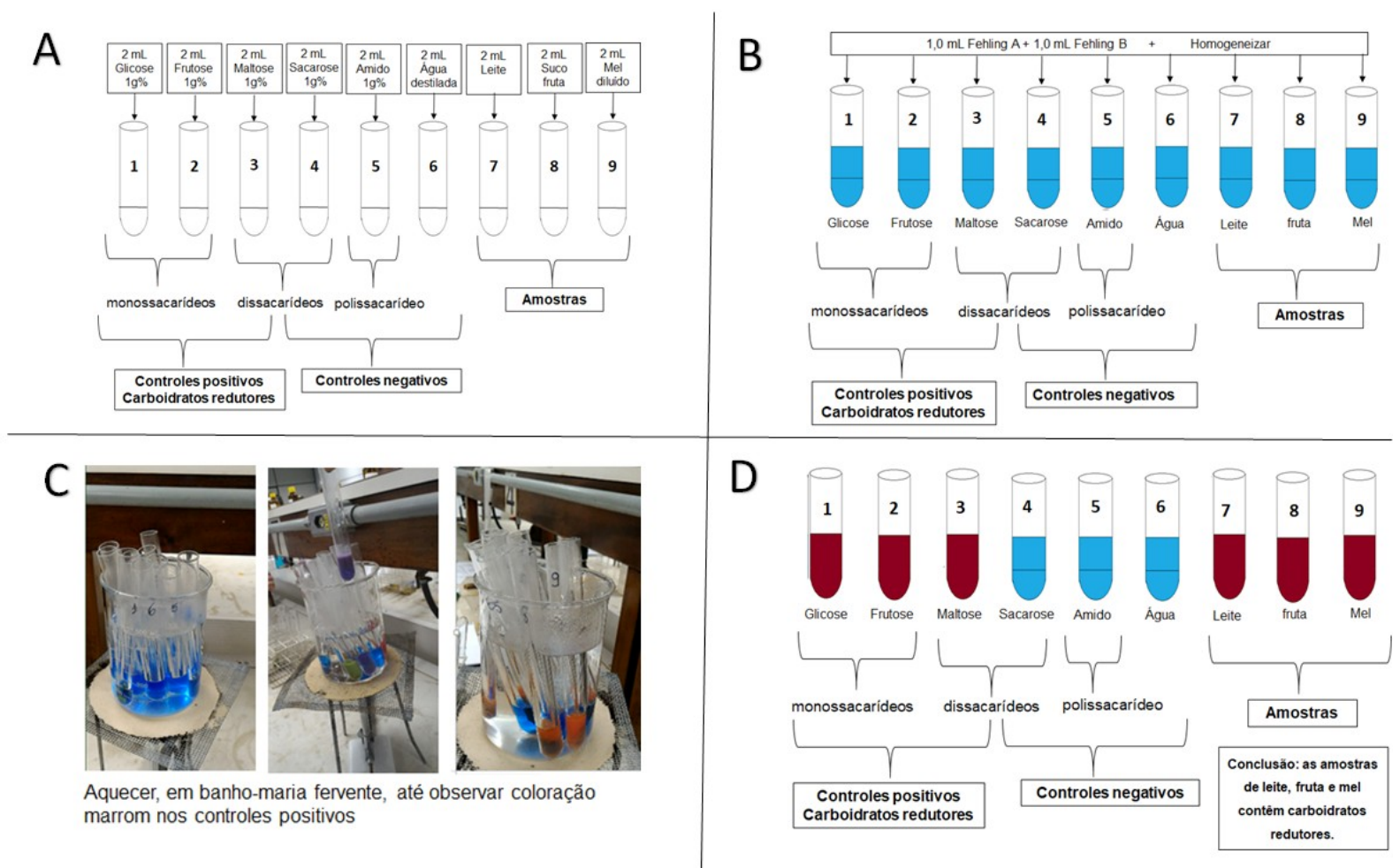

Figura 3: Reação de Fehling Fonte: autoria própria

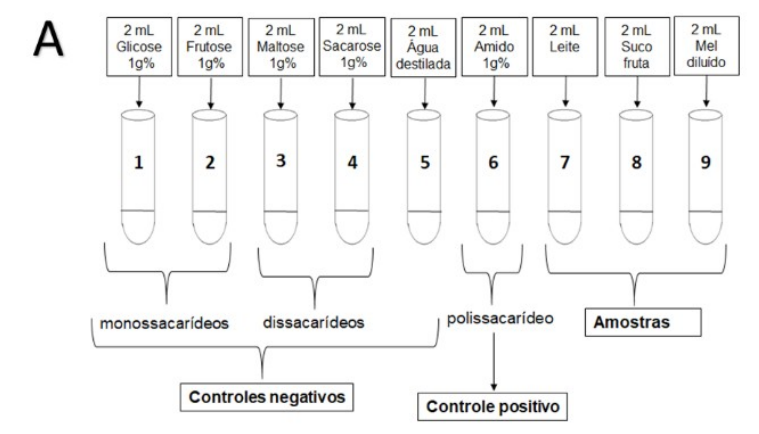

B
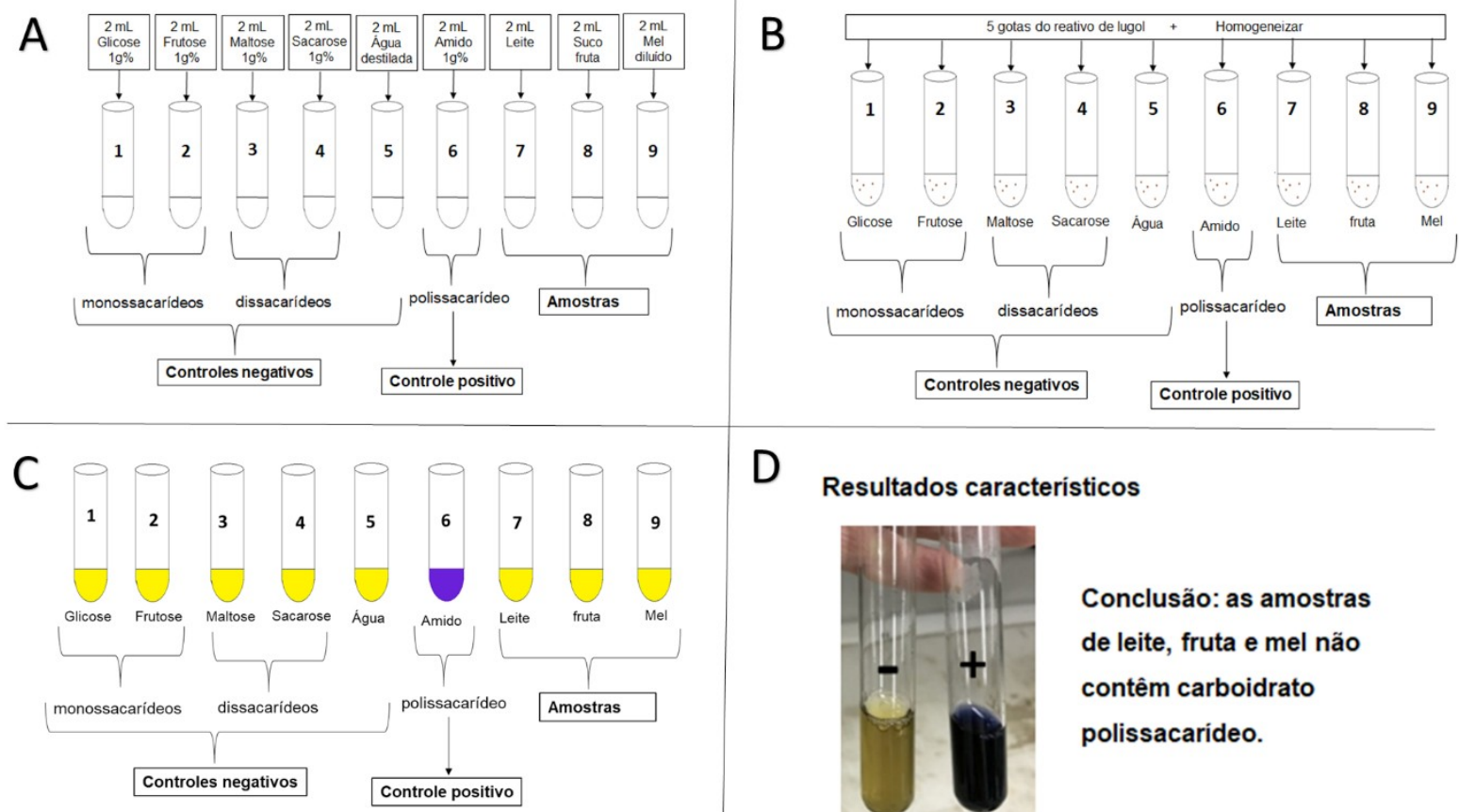

Desultados caracteristicos

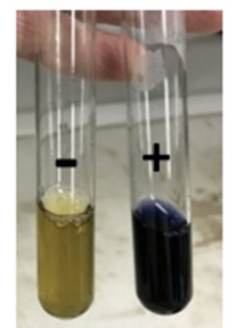

Conclusão: as amostras

de leite, fruta e mel não

contêm carboidrato

polissacarideo.

Figura 4: Reação de Lugol Fonte: autoria própria

Com a realização dos experimentos, mesmo na forma simulada, em aulas remotas, foi possível verificar que as amostras de leite, fruta e mel contêm carboidratos redutores e não contêm polissacarídeos. Também foi possível discutir e recuperar o conteúdo teórico de carboidratos. A aula na forma presencial enriqueceria o estudo do tema; por ser 
mais atrativa e possibilitar o desenvolvimento das habilidades específicas de laboratório de Bioquímica.

\section{Referências}

[1] Nelson DL, Cox MM. Princípios de Bioquímica de Lehninger. 7. ed. Porto Alegre: Artmed; 2018.

[2] Gropper SS, Smith JL, Groff JL. Nutrição avançada e metabolismo humano. 1. ed. São Paulo: Cengage Learning; 2011.

[3] Cisternas JR, Monte O, Montor WR. Fundamentos teóricos e práticos em Bioquímica. 1. ed. São Paulo: Atheneu; 2011.

[4] Cisternas JR, Varga J, Monte O. Fundamentos de Bioquímica Experimental. 1. ed. São Paulo: Atheneu; 1997.

[5] Bernardes, CF. Bioquímica Experimental. Campinas, 2018. [Apostila da Disciplina de Bioquímica - Curso de Química - PUC-Campinas]. 


\section{Apêndice A}

Text (Arial, 12, bold, justified, first line $1.25 \mathrm{~cm}$, paragraph $1.5 \mathrm{~cm}$. )

You should be entered as Appendix figures, texts, tables etc. that were used in the work and supplement the article, since be production of authors.

\section{Anexo A}

Text (Arial, 12, bold, left, first line $1.25 \mathrm{~cm}$, paragraph $1.5 \mathrm{~cm}$. )

You must be entered as an attachment figures, texts, tables etc. that were used in the work and supplement the article, not being production of the authors. 